\title{
Impulsive control for synchronizing delayed discrete complex networks with switching topology
}

\author{
Chaojie Li • David Y. Gao · Chao Liu • \\ Guo Chen
}

Received: 20 June 2013 / Accepted: 29 July 2013/Published online: 24 August 2013

(C) The Author(s) 2013. This article is published with open access at Springerlink.com

\begin{abstract}
In this paper, global exponential synchronization of a class of discrete delayed complex networks with switching topology has been investigated by using Lyapunov-Ruzimiki method. The impulsive scheme is designed to work at the time instant of switching occurrence. A time-varying delay-dependent criterion for impulsive synchronization is given to ensure the delayed discrete complex networks switching topology tending to a synchronous state. Furthermore, a numerical simulation is given to illustrate the effectiveness of main results
\end{abstract}

Keywords Impulsive synchronization - Complex networks $\cdot$ Switching topology $\cdot$ Lyapunov-Ruzimiki method

\section{Introduction}

It has long been understood that many physical, social, biological, and technological networks are modeled by a graph with non-trivial topological features. In this model, every node is an individual element of the whole system with certain pattern of connections, in which connections

C. Li $(\square) \cdot$ D. Y. Gao

School of Science, Information Technology and Engineering,

University of Ballarat, Mt Helen, VIC 3350, Australia

e-mail: cjlee.cqu@163.com

C. Liu

School of Electrical and Information Engineering,

Chongqing University of Science and Technology,

Chongqing 401331, People's Republic of China

G. Chen

School of Electrical and Information Engineering,

The University of Sydney, Sydney 2006, Australia between each pair of nodes are neither entirely regular nor entirely random [1-3]. These features do not occur in the mathematical models of networks that have been studied in the past, such as lattices or random graphs, but they do truly exist in nature. At present, derivatives of network science have been successfully applied to the analysis of metabolic and genetic regulatory networks, the design of robust and scalable communication networks both wired and wireless, the development of vaccination strategies for the control of disease, and a broad range of other practical issues.

Secure communication $[4,5]$, parallel image processing [6], and chemical reaction implemented by coupled chaotic systems have been an active research field during the last two decades. Synchronization issues are fundamentally important for any dynamical networks, and there is no exception for complex networks. As a consequence, theory and methods for synchronization of different families of complex networks have been extensively studied by many researchers (such as [7-15], and references therein). Network synchronization has been discussed in terms of spectral and statistical properties by authors [16]. The results on complete synchronization, phase synchronization, space lag synchronization, and cluster reflect the ideas of [17-19]. The improvement on different regimes of synchronization of discrete complex networks is abstracted from papers authored by [9, 20]. Some general cases of synchronization of complex networks with switching topology can be found in the literatures of $[15,21]$. From another angle, several approaches to synchronize a complex network have been proposed. Adaptive synchronization, impulsive synchronization scheme, and pining control synchronization have been considered by authors in [10-12, 22, 23-27]. In addition, systems with delays and multiplicative noises 
have been studied by considering $\mathrm{H}$-infinity method in [28] and [29].

However, all previous studies have limitations on synchronizing a state delayed discrete complex network. As known, connections between each pair of nodes in a complex network always change. The mutable topology can pose a significant threat on the global dynamical property of the whole networks. Indeed, the fact has been ignored by most of pioneering work. Additionally, the large time-varying delays may exist in switching topology which means data communication may occur in different sub-networks. It is much more complicated than previous studies. If the mutable topology and a large time-varying delay occur simultaneously in the discrete complex networks, it would be difficult to employ previous synchronization control schemes. Therefore, it is necessary to investigate new synchronization method.

Impulsive control has been successfully used to stabilize and synchronize dynamical systems, for examples, [3037]. And impulsive control technique could be an efficient method when a discrete change behavior is needed. The adjustment interest rate could agree with that. In this paper, we proposed an impulsive synchronization scheme for a state delayed discrete complex network with switching topology. For this control scheme, we consider that the impulsive control signal is designed to be input into all of nodes. Meanwhile, every time instant of impulsive effects occur precisely at the time instant of switching happening. In other words, when the complex networks switch its state at every instant of time, there is no delay between the controller functioning.

The paper is organized as follows. Section 2 presents some mathematical preliminaries needed in this work, and a generalized mathematical model for delayed discrete complex networks with switching topology. The main theorem for global synchronization of this type of discrete complex networks is then given in Sect. 3. In Sect. 4, a small-world network with 3 sub-networks involving 30 nodes is constructed to illustrate the effectiveness of our result. Section 5 concludes the paper.

\section{Preliminary}

First, we need to introduce some notations and definitions for the sake of exploring our main results. Let $\|\bullet\|$ denote the Euclidean norm; $\mathbb{R}^{n}$ denotes the $n$ dimensional Euclidean space, the set of natural numbers $\mathbb{N}=\{0,1,2, \ldots\}$, and, for certain positive integer $\tau$, we let $\mathbb{Z}_{-\tau}=\{-\tau,-\tau+1, \ldots, 0\}$. The family of $N$ linearly coupled discrete complex networks, consisting of time delay with respect to its system state and the switched topology, can be described by

$$
\begin{gathered}
x_{i}(k+1)=A x_{i}(k)+B f\left(x_{i}(k)\right)+D f\left(x_{i}(k-\tau(k))\right)+I(k) \\
+\sum_{j=1}^{N} c_{i j, \sigma(k)} \Gamma x_{j}(k-\tau(k)), \\
i=1,2, \ldots, N, k \in \mathbb{N} \\
x_{i k_{0}}=\phi(\theta), \quad \theta \in \mathbb{Z}_{-\tau},
\end{gathered}
$$

where $x_{i}(k)=\left(x_{i, 1}, x_{i, 2}, \ldots, x_{i, n}\right) \in \mathbb{R}^{n}$ represents the state vector of the $i$-th node at every instant of time $k$ and $n$ denotes the number of nodes affiliated to each subnetwork. $\mathbf{A} \in \mathbb{R}^{n \times n}, \mathbf{B} \in \mathbb{R}^{n \times n}$ and $\mathbf{D} \in \mathbb{R}^{n \times n}$ are known real matrices. $\mathbf{f}\left(\mathbf{x}_{\mathbf{i}}(\mathbf{k})\right)=\left(\mathbf{f}_{\mathbf{1}}\left(\mathbf{x}_{\mathbf{i}, \mathbf{1}}(\mathbf{k})\right), \mathbf{f}_{\mathbf{2}}\left(\mathbf{x}_{\mathbf{i}, \mathbf{2}}(\mathbf{k})\right), \ldots, \mathbf{f}_{\mathbf{n}}\left(\mathbf{x}_{\mathbf{i}, \mathbf{n}}(\mathbf{k})\right)\right)^{T}$ and $\mathbf{f}(\bullet): \mathbb{R}^{n} \longrightarrow \mathbb{R}^{n}$ is a smooth nonlinear vector-valued functions. $\mathbf{I}(\mathbf{k})=\left(I_{1}(k), I_{2}(k), \ldots, I_{n}(k)\right)^{T}$ is a n-dimensional vector from external input. $\mathbf{S}$ is a finite index set of $r$ elements: $\mathbf{S}=\left\{s_{1}, s_{2}, \ldots, s_{r}\right\}$. Let the switching function be denoted by $\sigma(k): \mathbb{N} \longrightarrow \mathbf{S}$, which is the switching signal from sudden changing of system dynamic without jumps in the state $\mathbf{x}$ at any switching instant. Specifically, we consider that it is a piecewise constant function and continuous from the right, indicating certain active subsystem regime, at every instant of time $k$ the index $\sigma(k)=s_{k} \in \mathbf{S}$; meanwhile, let the switching instants of $\sigma$ be denoted by $k_{m, x}(m=1,2, \ldots)$ and let $k_{0, x}:=0$. $C_{s_{k}}=\left(c_{i j, s_{k}}\right) \in \mathbb{Z}^{N \times N}$ represents the outer coupling configuration symmetric matrix defined as follows: For each active sub-system regime $s_{k}$, if there is a connection from node $j$ to node $i \quad(j \neq i)$, then $c_{i j, s_{k}}=c_{j i, s_{k}}>0$; otherwise $c_{i j, s_{k}}=c_{j i, s_{k}}=0$. Assume that

$c_{i i, s_{k}}=-\sum_{j=1, j \neq i}^{N} c_{i j, s_{k}}=-\sum_{j=1, j \neq i}^{N} c_{j i, s_{k}}, i \in N, s_{k} \in \mathbf{S}$.

The notation $\Gamma \in \mathbb{R}^{n \times n}$ represents the diagonal inner coupling matrix between two connected nodes. $\tau(k)$ is a time-varying delay with respect to each instant of time $k$ and satisfies $\tau(k) \in \mathbb{Z}_{-\tau} . \phi(\bullet): \mathbb{Z}_{-\tau} \longrightarrow \mathbb{R}^{n \times N}$ is smooth everywhere except at a finite number of points. The norm of $\phi(\bullet)$ is defined by $\|\phi(\theta)\|_{\tau}=\sup _{\theta \in \mathbb{Z}_{-\tau}}\{\|\phi(\theta)\|\}$.

In order to design an impulsive control scheme to synchronize system (1), we consider the evolutionary state is abruptly jumping at every impulsive instant of time $k_{u}$ from its open-loop state, which can be formulated by

$\Delta x_{i}\left(k_{m, u}\right)=J_{u} x_{i}^{*}\left(k_{m, u}\right), \quad m=1,2, \ldots \mathbb{N}$

where $x_{i}^{*}\left(k_{m, u}\right)$ stands for the primal state at time instant $k_{m, u}$ without impulsive jump. As usual, every impulsive instant of time $k_{l, u}$ satisfies $0=k_{0, u}<k_{1, u}<k_{2, u}<\cdots<$ $k_{m, u}<k_{m+1, u}<\ldots$ and $\lim _{m \rightarrow \infty} k_{m, u}=\infty ; J_{u}: \mathbb{R}^{n} \rightarrow \mathbb{R}^{n}$ $(m=1,2, \ldots)$ represents the impulsive jump strength. Therefore, at every impulsive instant of time $k_{m, u}$, the coupled states $x_{i}(k)-x_{j}(k)$ between connected node $i$ and $j$ can be described by 


$$
\begin{aligned}
x_{i}\left(k_{m, u}\right)-x_{j}\left(k_{m, u}\right)= & x_{i}^{*}\left(k_{m, u}\right)-x_{j}^{*}\left(k_{m, u}\right) \\
& +J_{u}\left[x_{i}^{*}\left(k_{m, u}\right)-x_{j}^{*}\left(k_{m, u}\right)\right] .
\end{aligned}
$$

Intuitively, a family of impulsive controller can be designed as

$$
U_{i}\left(k, x_{i}(k)\right)=\sum_{u=1}^{\infty} \delta\left(k-k_{m, u}\right) J_{u}\left(x_{i}^{*}\left(k_{m, u}\right)\right), \quad m=1,2, \ldots \mathbb{N}
$$

where $U_{i}\left(k, x_{i}(k)\right)$ represents a class of impulsive controller at each instant of time $k_{m, u} ; \delta(\bullet)$ denotes the Dirac discrete-time function. Correspondingly, by virtue of impulsive controller, the closed-loop discrete complex networks can be derived by the following form:

$$
\begin{gathered}
x_{i}(k+1)=A x_{i}(k)+B f\left(x_{i}(k)\right)+D f\left(x_{i}(k-\tau(k))\right)+I(k) \\
+\sum_{j=1}^{N} c_{i j, \sigma(k)} \Gamma x_{j}(k-\tau(k))+U_{i}\left(k+1, x_{i}^{*}(k+1)\right), \\
i=1,2, \ldots, N . \\
U_{i}\left(k+1, x_{i}^{*}(k+1)\right)=\sum_{u=1}^{\infty} \delta\left(k+1-k_{m, u}\right) J_{u}\left[A x_{i}(k)\right. \\
+B f\left(x_{i}(k)\right)+D f\left(x_{i}(k-\tau(k))\right) \\
\left.+I(k)+\sum_{j=1}^{N} c_{i j, \sigma(k)} \Gamma x_{j}(k-\tau(k))\right], \\
m=1,2, \ldots, \mathbb{N} .
\end{gathered}
$$

Assumption 1 For each nonlinear function $\mathbf{f}_{\mathbf{i}}(\bullet)$ $(i=1,2, \ldots, n)$, suppose that it is globally Lipschitz continues function and satisfies

$\left\|f_{i}\left(x_{1}\right)-f_{i}\left(x_{2}\right)\right\| \leq \hat{l}_{i}\left\|x_{1}-x_{2}\right\|, \quad i=1,2, \ldots, n, \forall x_{1}, x_{2} \in \mathbb{R}$,

where $\hat{l}_{i}$ is certain positive constant.

Definition 1 The system of the impulsive controlled discrete complex networks (7) is said to be globally exponentially synchronized, if for any initial condition $\phi(\bullet): \mathbb{Z}_{-\tau} \rightarrow \mathbb{R}^{n \times N}$, and there exist two positive constants $\lambda$ and $M_{0} \geq 1$ such that

$$
\left\|x_{i}(k)-x_{j}(k)\right\| \leq M_{0} \mathrm{e}^{-\lambda\left(k-k_{0}\right)}, \quad 1 \leq i \leq j \leq N
$$

holds for all $k>k_{0}$.

Lemma [18] Let $\mathbf{W}=\left(w_{i j}\right)_{N \times N}, \mathbf{P} \in \mathbb{R}^{n \times n}, \mathbf{x}=$ $\left(x_{1}, x_{2}, \ldots, x_{N}\right)^{T}$ and $\mathbf{y}=\left(y_{1}, y_{2}, \ldots, y_{N}\right)^{T}$ with $x_{k}, y_{k} \in$ $\mathbb{R}^{N}(k=1,2, \ldots, N)$. If $\mathbf{W}=\mathbf{W}^{T}$ and each row sum of $\mathbf{W}$ is zero, then
$\mathbf{x}^{\mathbf{T}}(\mathbf{W} \otimes \mathbf{P}) \mathbf{y}=-\sum_{i=1}^{N-1} \sum_{j=i+1}^{N} w_{i j}\left(x_{i}-x_{j}\right)^{T} P\left(y_{i}-y_{j}\right)$.

\section{Main results}

When the impulsive controller can be functioning simultaneously at the state of discrete complex networks' switching signal, the equivalent impulsive controlled system is rewritten by using the matrix Kronecker product (see [38])

$$
\begin{aligned}
x(k+1)= & \left(I_{N} \otimes A\right) x(k)+\left(I_{N} \otimes B\right) F(x(k)) \\
& +\left(I_{N} \otimes D\right) F(x(k-\tau))+I(k) \\
& +\left(C_{\sigma(k)} \otimes \Gamma\right) x(k-\tau), \quad k \neq k_{m, u} \\
x\left(k_{m, u}\right)=[ & \left.I_{N} \otimes\left(I_{N}+J_{u}\left(k_{m, u}\right)\right)\right] x\left(k_{m, u}-1\right),
\end{aligned}
$$

for any $k, m \in \mathbb{N}$.

Theorem 1 Under Assumption 1., the impulsive controlled complex network (12) is exponentially synchronized if there exist certain positive integer $m_{\tau}$, positive scalars $\varepsilon_{\sigma(k)}, p_{\sigma(k)}, q_{\sigma(k)}$, and positive-definite matrices $P_{\sigma(k)} \in \mathbb{R}^{n \times n}, Q_{l, \sigma(k)} \in \mathbb{R}^{n \times n}(l=1,2, \ldots 6)$ such that

- Given $\mu \geq 1$ and $P_{\sigma\left(k_{m, x}\right)} \leq \mu P_{\sigma\left(k_{m+1, x}\right)}$, for any $k \in\left[k_{m, x}, k_{m+1, x}-1\right]$ in corresponding sub-state $\sigma\left(k_{m, x}\right)$,

$p_{\sigma\left(k_{m, x}\right)}-\left[\frac{\lambda_{\max }\left(\Pi_{\sigma\left(k_{m, x}\right)}\right)}{\lambda_{\min }\left(P_{\sigma\left(k_{m, x}\right)}^{-1}\right)}+\mu q_{\sigma\left(k_{m, x}\right)} \frac{\lambda_{\max }\left(\Omega_{\sigma\left(k_{m, x}\right)}\right)}{\lambda_{\min }\left(P_{\sigma\left(k_{m-m_{x}, x}\right)}^{-1}\right)}\right] \geq 0$,

where

$$
\begin{aligned}
& \Pi_{\sigma\left(k_{m, x}\right)}=A^{T} P_{\sigma\left(k_{m, x}\right)} A+L^{T} B^{T} P_{\sigma\left(k_{m, x}\right)} B L \\
& +L^{T} B^{T} P_{\sigma\left(k_{m, x}\right)}^{T} Q_{1, \sigma\left(k_{m, x}\right)} P_{\sigma\left(k_{m, x}\right)} B L \\
& +A^{T} Q_{1, \sigma\left(k_{m, x}\right)} A+A^{T} Q_{2, \sigma\left(k_{m, x}\right)}^{-1} A \\
& -N C_{\sigma\left(k_{m, x}\right)} A^{T} Q_{3, \sigma\left(k_{m, x}\right)}^{-1} A C_{\sigma\left(k_{m, x}\right)} \\
& -L^{T} N C_{\sigma\left(k_{m, x}\right)} B^{T} Q_{5, \sigma\left(k_{m, x}\right)} B C_{\sigma\left(k_{m, x}\right)} L \\
& +L^{T} B^{T} Q_{4, \sigma\left(k_{m, x}\right)} B L \\
& \Omega_{\sigma\left(k_{m, x}\right)}=L^{T} D^{T} P_{\sigma\left(k_{m, x}\right)} D L-N C_{\sigma\left(k_{m, x}\right)}^{2} \Gamma^{T} P_{\sigma\left(k_{m, x}\right)} \Gamma \\
& +L^{T} D^{T} P_{\sigma\left(k_{m, x}\right)}^{T} Q_{2, \sigma\left(k_{m, x}\right)} P_{\sigma\left(k_{m, x}\right)} D L \\
& -\Gamma^{T} P_{\sigma\left(k_{m, x}\right)}^{T} Q_{3, \sigma\left(k_{m, x}\right)} P_{\sigma\left(k_{m, x}\right)} \Gamma \\
& +L^{T} D^{T} P_{\sigma\left(k_{m, x}\right)}^{T} Q_{4, \sigma\left(k_{m, x}\right)} P_{\sigma\left(k_{m, x}\right)} D L \\
& \text { - } \Gamma^{T} P_{\sigma\left(k_{m, x}\right)}^{T} Q_{5, \sigma\left(k_{m, x}\right)} P_{\sigma\left(k_{m, x}\right)} \Gamma \\
& -L^{T} N C_{\sigma\left(k_{m, x}\right)} D^{T} Q_{6, \sigma\left(k_{m, x}\right)}^{-1} D C_{\sigma\left(k_{m, x}\right)} L \\
& -\Gamma^{T} P_{\sigma\left(k_{m, x}\right)}^{T} Q_{6, \sigma\left(k_{m, x}\right)} P_{\sigma\left(k_{m, x}\right)} \Gamma \text {. } \\
& \text { - } \mu \lambda_{\max }^{2}\left(1+J_{u}\left(k_{m, x}\right)\right)<\mathrm{e}^{\varepsilon_{\sigma\left(k_{m, x}\right)}\left(k_{m+1, x}-k_{m, x}\right)} \text {. } \\
& \text { - } q_{\sigma\left(k_{m, x}\right)} \geq \mathrm{e}^{\varepsilon_{\sigma\left(k_{m, x}\right)}\left(k_{m+1, x}-k_{m, x}+1\right)+\sum_{i=0}^{m_{\tau}-1} \varepsilon_{k_{m-i, x}}\left(k_{m+1-i, x}-k_{m-i, x}\right)} \text {, } \\
& \text { where } m_{\tau}=\left\lceil\frac{\tau}{\inf \left\{k_{m, x}-k_{m-1, x}\right\}}\right\rceil \text {. }
\end{aligned}
$$

Proof Consider the following Lyapunov function:

$V(k)=x^{T}(k)\left(W \otimes P_{\sigma(k)}\right) x(k)$, 
for any $k \in\left[k_{m, x}, k_{m+1, x}-1\right], \quad m=1,2, \ldots$ where

$W=\left[\begin{array}{cccc}N-1 & -1 & \ldots & -1 \\ -1 & N-1 & \ldots & -1 \\ \ldots & \ldots & \ldots & \ldots \\ -1 & -1 & \ldots & N-1\end{array}\right]$.

Let $\varphi\left(\left\|\phi_{i}(\theta)-\phi_{j}(\theta)\right\|^{2}\right)=\lambda_{\max }\left(P_{\sigma(\theta)}\right)\left\|\phi_{i}(\theta)-\phi_{j}(\theta)\right\|^{2}$, thus for the case $k \in \mathbb{Z}_{\tau}$,

$$
\begin{aligned}
V(\theta) & =x^{T}(\theta)\left(W \otimes P_{\sigma(0)}\right) x(\theta) \\
& =\sum_{i=1}^{N-1} \sum_{j=i+1}^{N}\left(x_{i}(\theta)-x_{j}(\theta)\right)^{T} P_{\sigma(0)}\left(x_{i}(\theta)-x_{j}(\theta)\right) \\
& \leq \varphi\left(\left\|\phi_{i}(\theta)-\phi_{j}(\theta)\right\|_{\tau}^{2}\right) \\
& =\varphi\left(\|\phi(\theta)\|_{\tau}^{2}\right) .
\end{aligned}
$$

Choose $M \geq 1$, such that

$$
\begin{aligned}
\varphi\left(\|\phi(\theta)\|_{\tau}^{2}\right) & \leq M \varphi\left(\|\phi(\theta)\|_{\tau}^{2}\right) \mathrm{e}^{-\lambda\left(k_{1, x}-k_{0, x}\right)} \mathrm{e}^{-\varepsilon_{\sigma\left(k_{0, x}\right)}\left(k_{1, x}-k_{0, x}\right)} \\
& <M \varphi\left(\|\phi(\theta)\|_{\tau}^{2}\right) \mathrm{e}^{-\lambda\left(k_{1, x}-k_{0, x}\right)} \\
& <q_{\sigma\left(k_{0, x}\right)} \varphi\left(\|\phi(\theta)\|_{\tau}^{2}\right)
\end{aligned}
$$

By claiming that

$V(k) \leq M \varphi\left(\|\phi(\theta)\|_{\tau}^{2}\right) \mathrm{e}^{-\lambda\left(k_{m, x}-k_{0, x}\right)}, k \in\left[k_{m-1, x}, k_{m, x}-1\right]$, $m \in N$.

We first need to show that

$V(k) \leq M \varphi\left(\|\phi(\theta)\|_{\tau}^{2}\right) \mathrm{e}^{-\lambda\left(k_{1, x}-k_{0, x}\right)}, k \in\left[k_{0, x}, k_{1, x}-1\right]$.

Obviously, for $k \in\left[k_{0, x}-\tau, k_{0, x}-1\right]$,

$$
\begin{aligned}
V(k) & \leq \varphi\left(\|\phi(\theta)\|_{\tau}^{2}\right) \\
& \leq M \varphi\left(\|\phi(\theta)\|_{\tau}^{2}\right) \mathrm{e}^{-\lambda\left(k_{1, x}-k_{0, x}\right)} \mathrm{e}^{-\varepsilon_{\sigma\left(k_{0, x}\right)}\left(k_{1, x}-k_{0, x}\right)} .
\end{aligned}
$$

If (19) is not true, then there exists certain instant of time $\bar{k} \in\left[k_{0, x}, k_{1, x}-1\right]$ satisfying

$\bar{k}=\min \left\{k \in\left[k_{0, x}, k_{1, x}-1\right]: V(k)>M \varphi\left(\|\phi(\theta)\|_{\tau}^{2}\right) \mathrm{e}^{-\lambda\left(k_{1, x}-k_{0, x}\right)}\right\}$

such that

$$
\begin{aligned}
V(\bar{k}) & >M \varphi\left(\|\phi(\theta)\|_{\tau}^{2}\right) \mathrm{e}^{-\lambda\left(k_{1, x}-k_{0, x}\right)} \\
& >M \varphi\left(\|\phi(\theta)\|_{\tau}^{2}\right) \mathrm{e}^{-\lambda\left(k_{1, x}-k_{0, x}\right)} \mathrm{e}^{-\varepsilon_{\sigma\left(k_{0, x}\right)}\left(k_{1, x}-k_{0, x}\right)} \\
& >\varphi\left(\|\phi(\theta)\|_{\tau}^{2}\right)
\end{aligned}
$$

and

$$
V(k) \leq M \varphi\left(\|\phi(\theta)\|_{\tau}^{2}\right) \mathrm{e}^{-\lambda\left(k_{1, x}-k_{0, x}\right)}, k \in\left[k_{0, x}-\tau, \bar{k}-1\right]
$$

Considering that

$$
\begin{aligned}
k^{*}= & \max \left\{k \in\left[k_{0, x}, \bar{k}\right]: V(k) \leq M \varphi\left(\|\phi(\theta)\|_{\tau}^{2}\right)\right. \\
& \left.\mathrm{e}^{-\lambda\left(k_{1, x}-k_{0, x}\right)} \mathrm{e}^{-\varepsilon_{\sigma\left(k_{0, x}\right)}\left(k_{1, x}-k_{0, x}\right)}\right\},
\end{aligned}
$$

from which we have

$$
\begin{gathered}
V(k)>M \varphi\left(\|\phi(\theta)\|_{\tau}^{2}\right) \mathrm{e}^{-\lambda\left(k_{1, x}-k_{0, x}\right)} \mathrm{e}^{-\varepsilon_{\sigma\left(k_{0, x}\right)}\left(k_{1, x}-k_{0, x}\right)}, \\
k \in\left[k^{*}+1, \bar{k}-1\right] .
\end{gathered}
$$

Therefore,

$V\left(k^{*}\right) \leq V(k) \leq V(\bar{k}), k \in\left[k^{*}, \bar{k}\right]$.

And for any $k \in\left[k^{*}, \bar{k}-1\right]$,

$$
\begin{aligned}
V(k+s) & \leq M \varphi\left(\|\phi(\theta)\|_{\tau}^{2}\right) \mathrm{e}^{-\lambda\left(k_{1, x}-k_{0, x}\right)} \\
& \leq \mathrm{e}^{\varepsilon_{\sigma\left(k_{0, x}\right)}\left(k_{1, x}-k_{0, x}\right)} V\left(k^{*}\right) \\
& \leq q_{\sigma\left(k_{0, x}\right)} V(k) .
\end{aligned}
$$

Consider the increment of $V(k)$ along the solution of discrete complex networks (12) in the interval $k \in\left[k^{*}, \bar{k}-1\right]$, one observes

$$
\begin{aligned}
& \Delta V(k)=V(k+1)-V(k) \\
& =\left[\left(I_{N} \otimes A\right) x(k)+\left(I_{N} \otimes B\right) F(x(k))\right. \\
& +\left(I_{N} \otimes D\right) F(x(k-\tau))+I(k) \\
& \left.+\left(C_{\sigma(k)} \otimes \Gamma\right) x(k-\tau)\right]^{T}\left(W \otimes P_{\sigma(k+1)}\right)\left[\left(I_{N} \otimes A\right) x(k)\right. \\
& +\left(I_{N} \otimes B\right) F(x(k))+\left(I_{N} \otimes D\right) F(x(k-\tau)) \\
& \left.+I(k)+\left(C_{\sigma(k)} \otimes \Gamma\right) x(k-\tau)\right]-x^{T}(k) P_{\sigma(k)} x(k) \\
& =\left[\left(I_{N} \otimes A\right) x(k)+\left(I_{N} \otimes B\right) F(x(k))\right. \\
& +\left(I_{N} \otimes D\right) F(x(k-\tau))+I(k) \\
& \left.+\left(C_{\sigma(k)} \otimes \Gamma\right) x(k-\tau)\right]^{T}\left(W \otimes P_{\sigma\left(k_{0, x}\right)}\right)\left[\left(I_{N} \otimes A\right) x(k)\right. \\
& +\left(I_{N} \otimes B\right) F(x(k))+\left(I_{N} \otimes D\right) F(x(k-\tau)) \\
& \left.+I(k)+\left(C_{\sigma(k)} \otimes \Gamma\right) x(k-\tau)\right]-x^{T}(k) P_{\sigma\left(k_{0 . x}\right)} x(k) \\
& =x^{T}(k)\left(I_{N} \otimes A^{T}\right)\left(W \otimes P_{\sigma\left(k_{0, x}\right)}\right)\left(I_{N} \otimes A\right) x(k) \\
& +F^{T}(x(k))\left(I_{N} \otimes B^{T}\right)\left(W \otimes P_{\sigma\left(k_{0, x}\right)}\right)\left(I_{N} \otimes B\right) F(x(k)) \\
& +F^{T}(x(k-\tau))\left(I_{N} \otimes D^{T}\right)\left(W \otimes P_{\sigma\left(k_{0, x}\right)}\right)\left(I_{N} \otimes D\right) F(x(k-\tau)) \\
& +I^{T}(k)\left(W \otimes P_{\sigma\left(k_{0, x}\right)}\right) I(k) \\
& +x^{T}(k-\tau)\left(C_{\sigma\left(k_{0, x}\right)}^{T} \otimes \Gamma^{T}\right)\left(W \otimes P_{\sigma\left(k_{0, x}\right)}\right)\left(C_{\sigma\left(k_{0, x}\right)} \otimes \Gamma\right) x(k-\tau) \\
& +2 x^{T}(k)\left(I_{N} \otimes A^{T}\right)\left(W \otimes P_{\sigma\left(k_{0, x}\right)}\right)\left(I_{N} \otimes B\right) F(x(k)) \\
& +2 x^{T}(k)\left(I_{N} \otimes A^{T}\right)\left(W \otimes P_{\sigma\left(k_{0, x}\right)}\right)\left(I_{N} \otimes D\right) F(x(k-\tau)) \\
& +2 x^{T}(k)\left(I_{N} \otimes A^{T}\right)\left(W \otimes P_{\sigma\left(k_{0 . x}\right)}\right) I(k) \\
& +2 x^{T}(k)\left(I_{N} \otimes A^{T}\right)\left(W \otimes P_{\sigma\left(k_{0, x}\right)}\right)\left(C_{\sigma\left(k_{0, x}\right)} \otimes \Gamma\right) x(k-\tau) \\
& +2 F^{T}(x(k))\left(I_{N} \otimes B^{T}\right)\left(W \otimes P_{\sigma\left(k_{0, x}\right)}\right)\left(I_{N} \otimes D\right) F(x(k-\tau)) \\
& +2 F^{T}(x(k))\left(I_{N} \otimes B^{T}\right)\left(W \otimes P_{\sigma\left(k_{0, x}\right)}\right) I(k) \\
& +2 F^{T}(x(k))\left(I_{N} \otimes B^{T}\right)\left(W \otimes P_{\sigma\left(k_{0, x}\right)}\right)\left(C_{\sigma\left(k_{0, x}\right)} \otimes \Gamma\right) x(k-\tau) \\
& +2 F^{T}(x(k))\left(I_{N} \otimes B^{T}\right)\left(W \otimes P_{\sigma\left(k_{0, x}\right)}\right)\left(I_{N} \otimes D\right) F(x(k-\tau)) \\
& +2 F^{T}(x(k))\left(I_{N} \otimes B^{T}\right)\left(W \otimes P_{\sigma\left(k_{0 . x}\right)}\right) I(k) \\
& +2 F^{T}(x(k))\left(I_{N} \otimes B^{T}\right)\left(W \otimes P_{\sigma\left(k_{0, x}\right)}\right)\left(C_{\sigma\left(k_{0, x}\right)} \otimes \Gamma\right) x(k-\tau) \\
& +2 F^{T}(x(k-\tau))\left(I_{N} \otimes D^{T}\right)\left(W \otimes P_{\sigma\left(k_{0, x}\right)}\right) I(k) \\
& +2 F^{T}(x(k-\tau))\left(I_{N} \otimes D^{T}\right)\left(W \otimes P_{\sigma\left(k_{0, x}\right)}\right)\left(C_{\sigma\left(k_{0, x}\right)} \otimes \Gamma\right) x(k-\tau) \\
& +2 I^{T}(k)\left(W \otimes P_{\sigma\left(k_{0, x}\right)}\right)\left(C_{\sigma\left(k_{0, x}\right)} \otimes \Gamma\right) x(k-\tau) \\
& -x^{T}(k) P_{\sigma\left(k_{0, x}\right)} x(k)
\end{aligned}
$$

Note that

$\left(W \otimes P_{\sigma(k)}\right) I(k)=0 \quad$ and $\quad I^{T}(k)\left(W \otimes P_{\sigma(k)}\right)=0$. 
For the simplicity of calculation, we define

$C_{\sigma\left(k_{0, x}\right)} W C_{\sigma\left(k_{0, x}\right)}=N C_{\sigma\left(k_{0, x}\right)}^{2}$.

$W C_{\sigma\left(k_{0, x}\right)}=N C_{\sigma\left(k_{0, x}\right)}$.

$x_{i j}(k)=x_{i}(k)-x_{j}(k)$.

$x_{i j}(k-\tau)=x_{i}(k-\tau)-x_{j}(k-\tau)$.

$F_{i j}(x(k))=F_{i}(x(k))-F_{j}(x(k))$.

$F_{i j}(x(k-\tau))=F_{i}(x(k-\tau))-F_{j}(x(k-\tau))$.

According to Lemma 1, we can obtain that

$$
\begin{aligned}
& \Delta V(k)=V(k+1)-V(k) \\
& =\sum_{i=1}^{N-1} \sum_{j=i+1}^{N}\left[x_{i j}^{T}(k) A^{T} P_{\sigma\left(k_{0, x}\right)} A x_{i j}(k)\right. \\
& +x_{i j}^{T}(k) L^{T} B^{T} P_{\sigma\left(k_{0, x}\right)} B L x_{i j}(k) \\
& +x_{i j}^{T}(k-\tau) L^{T} D^{T} P_{\sigma\left(k_{0, x}\right)} D L x_{i j}(k-\tau) \\
& +x_{i j}^{T}(k-\tau) N C_{\sigma\left(k_{0, x}\right)}^{2} \Gamma^{T} P_{\sigma\left(k_{0, x}\right)} \Gamma x_{i j}(k-\tau) \\
& +x_{i j}^{T}(k) A^{T} Q_{1, \sigma\left(k_{0, x}\right)}^{-1} A x_{i j}(k) \\
& +x_{i j}^{T}(k) L^{T} B^{T} P_{\sigma\left(k_{0, x}\right)}^{T} Q_{1, \sigma\left(k_{0, x}\right)} P_{\sigma\left(k_{0, x}\right)} B L x_{i j}(k) \\
& +x_{i j}^{T}(k) A^{T} Q_{2, \sigma\left(k_{0, x}\right)}^{-1} A x_{i j}(k) \\
& +x_{i j}^{T}(k-\tau) L^{T} D^{T} P_{\sigma\left(k_{0, x}\right)}^{T} Q_{2, \sigma\left(k_{0, x}\right)} P_{\sigma\left(k_{0, x}\right)} D L x_{i j}(k-\tau) \\
& -x_{i j}^{T}(k) N C_{\sigma\left(k_{0, x}\right)} A^{T} Q_{3, \sigma\left(k_{0, x}\right)}^{-1} A C_{\sigma\left(k_{0, x}\right)} x_{i j}(k) \\
& -x_{i j}^{T}(k-\tau) \Gamma^{T} P_{\sigma\left(k_{0, x}\right)}^{T} Q_{3, \sigma\left(k_{0, x}\right)} P_{\sigma\left(k_{0, x}\right)} \Gamma x_{i j}(k-\tau) \\
& +x_{i j}^{T}(k) L^{T} B^{T} Q_{4, \sigma\left(k_{0, x}\right)}^{-1} B L x_{i j}(k) \\
& +x_{i j}^{T}(k-\tau) L^{T} D^{T} P_{\sigma\left(k_{0, x}\right)}^{T} Q_{4, \sigma\left(k_{0, x}\right)} P_{\sigma\left(k_{0, x}\right)} D L x_{i j}(k-\tau) \\
& -x_{i j}^{T}(k) L^{T} N C_{\sigma\left(k_{0, x}\right)} B^{T} Q_{5, \sigma\left(k_{0, x}\right)}^{-1} B C_{\sigma\left(k_{0, x}\right)} L x_{i j}(k) \\
& -x_{i j}^{T}(k-\tau) \Gamma^{T} P_{\sigma\left(k_{0, x}\right)}^{T} Q_{5, \sigma\left(k_{0, x}\right)} P_{\sigma\left(k_{0, x}\right)} \Gamma x_{i j}(k-\tau) \\
& -x_{i j}^{T}(k-\tau) L^{T} N C_{\sigma\left(k_{0, x}\right)} D^{T} Q_{6, \sigma\left(k_{0, x}\right)}^{-1} D C_{\sigma\left(k_{0, x}\right)} L x_{i j}(k-\tau) \\
& -x_{i j}^{T}(k-\tau) \Gamma^{T} P_{\sigma\left(k_{0, x}\right)}^{T} Q_{6, \sigma\left(k_{0, x}\right)} P_{\sigma\left(k_{0, x}\right)} \Gamma x_{i j}(k-\tau) \\
& \left.-x_{i j}^{T}(k) P_{\sigma\left(k_{0, x}\right)} x_{i j}(k)\right] \\
& =\sum_{i=1}^{N-1} \sum_{j=i+1}^{N}\left\{x _ { i j } ^ { T } ( k ) \left[A^{T} P_{\sigma\left(k_{0, x}\right)} A+A^{T} Q_{1, \sigma\left(k_{0, x}\right)} A\right.\right. \\
& +L^{T} B^{T} P_{\sigma\left(k_{0, x}\right)} B L+A^{T} Q_{2, \sigma\left(k_{m, x}\right)}^{-1} A \\
& +L^{T} B^{T} P_{\sigma\left(k_{m, x}\right)}^{T} Q_{1, \sigma\left(k_{m, x}\right)} P_{\sigma\left(k_{m, x}\right)} B L \\
& -N C_{i j, \sigma\left(k_{0, x}\right)} A^{T} Q_{3, \sigma\left(k_{0, x}\right)}^{-1} A C_{i j, \sigma\left(k_{0, x}\right)} \\
& +L^{T} B^{T} Q_{4, \sigma\left(k_{0, x}\right)} B L \\
& \left.-L^{T} N C_{\sigma\left(k_{0, x}\right)} B^{T} Q_{5, \sigma\left(k_{0, x}\right)} B C_{\sigma\left(k_{0, x}\right)} L-P_{\sigma\left(k_{0, x}\right)}\right] x_{i j}(k) \\
& +x_{i j}^{T}(k-\tau)\left[L^{T} D^{T} P_{\sigma\left(k_{0, x}\right)} D L-N C_{\sigma\left(k_{0, x}\right)}^{(2)} \Gamma^{T} P_{\sigma\left(k_{0, x}\right)} \Gamma\right. \\
& +L^{T} D^{T} P_{\sigma\left(k_{0, x}\right)}^{T} Q_{2, \sigma\left(k_{0, x}\right)} P_{\sigma\left(k_{0, x}\right)} D L \\
& -\Gamma^{T} P_{\sigma\left(k_{0, x}\right)}^{T} Q_{3, \sigma\left(k_{0, x}\right)} P_{\sigma\left(k_{0, x}\right)} \Gamma
\end{aligned}
$$

$$
\begin{aligned}
& +L^{T} D^{T} P_{\sigma\left(k_{0, x}\right)}^{T} Q_{4, \sigma\left(k_{0, x}\right)} P_{\sigma\left(k_{0, x}\right)} D L \\
& -\Gamma^{T} P_{\sigma\left(k_{0, x}\right)}^{T} Q_{5, \sigma\left(k_{0, x}\right)} P_{\sigma\left(k_{0, x}\right)} \Gamma \\
& -L^{T} N C_{\sigma\left(k_{0, x}\right)} D^{T} Q_{6, \sigma\left(k_{0, x}\right)}^{-1} D C_{\sigma\left(k_{0, x}\right)} L \\
& \left.\left.-\Gamma^{T} P_{\sigma\left(k_{0, x}\right)}^{T} Q_{6, \sigma\left(k_{0, x}\right)} P_{\sigma\left(k_{0, x}\right)} \Gamma\right] x_{i j}(k-\tau)\right\} \\
= & {\left[\frac{\lambda_{\max }\left(\Pi_{\sigma\left(k_{0, x}\right)}\right)}{\lambda_{\min }\left(P_{\sigma\left(k_{0, x}\right)}^{-1}\right)}-1\right] V(k)+\frac{\lambda_{\max }\left(\Omega_{\sigma\left(k_{0, x}\right)}\right)}{\lambda_{\min }\left(P_{\sigma\left(k_{0, x}\right)}^{-1}\right)} V(k-\tau) } \\
= & {\left[\frac{\lambda_{\max }\left(\Pi_{\sigma\left(k_{0, x}\right)}\right)}{\lambda_{\min }\left(P_{\sigma\left(k_{0, x}\right)}^{-1}\right)}+\mu q_{\sigma\left(k_{0, x}\right)} \frac{\lambda_{\max }\left(\Omega_{\sigma\left(k_{0, x}\right)}\right)}{\lambda_{\min }\left(P_{\sigma\left(k_{0, x}\right)}^{-1}\right)}-1\right] V(k) }
\end{aligned}
$$

Therefore, we obtain

$$
\begin{aligned}
V(\bar{k}) \leq & {\left[\frac{\lambda_{\max }\left(\Pi_{\sigma\left(k_{0, x}\right)}\right)}{\lambda_{\min }\left(P_{\sigma\left(k_{0, x}\right)}^{-1}\right)}+\mu q_{\sigma\left(k_{0, x}\right)} \frac{\lambda_{\max }\left(\Omega_{\sigma\left(k_{0, x}\right)}\right)}{\lambda_{\min }\left(P_{\sigma\left(k_{0, x}\right)}^{-1}\right)}\right] V(\bar{k}-1) } \\
& \leq\left[\frac{\lambda_{\max }\left(\Pi_{\sigma\left(k_{0, x}\right)}\right)}{\lambda_{\min }\left(P_{\sigma\left(k_{0, x}\right)}^{-1}\right)}+\mu q_{\sigma\left(k_{0, x}\right)} \frac{\lambda_{\max }\left(\Omega_{\sigma\left(k_{0, x}\right)}\right)}{\lambda_{\min }\left(P_{\sigma\left(k_{0, x}\right)}^{-1}\right)}\right] \\
& \leq\left[\frac{\lambda_{\max }\left(\Pi_{\sigma\left(k_{0, x}\right)}\right)}{\lambda_{\min }\left(P_{\sigma\left(k_{0, x}\right)}^{-1}\right)}+\mu q_{\sigma\left(k_{0, x}\right)} \frac{\lambda_{\max }\left(\Omega_{\sigma\left(k_{0, x}\right)}\right)}{\lambda_{\min }\left(P_{\sigma\left(k_{0, x}\right)}^{-1}\right)}\right]^{\left(k_{1, x}-k_{0, x}\right)} \\
& \cdot M \varphi\left(\|\phi(\theta)\|_{\tau}^{2}\right) \mathrm{e}^{-\lambda\left(k_{1, x}-k_{0, x}\right)} \mathrm{e}^{-\varepsilon_{\sigma\left(k_{0, x}\right)}\left(k_{1, x}-k_{0, x}\right)} \\
& \leq M \varphi\left(\|\phi(\theta)\|_{\tau}^{2}\right) \mathrm{e}^{-\lambda\left(k_{1, x}-k_{0, x}\right)} \\
& <V(\bar{k}) .
\end{aligned}
$$

We have a contradiction here and (19) is true. Next comes that we suppose the claim (18) holds for $\mathrm{m}=1,2, \ldots, \bar{m}$, such that

$V(k) \leq M \varphi\left(\|\phi(\theta)\|_{\tau}^{2}\right) \mathrm{e}^{-\lambda\left(k_{m, x}-k_{0, x}\right)}, k \in\left[k_{m-1, x}, k_{m, x}-1\right]$.

Correspondingly, we should prove the Eq. (18) holds for $\mathrm{m}=\bar{m}+1$,

$V(k) \leq M \varphi\left(\|\phi(\theta)\|_{\tau}^{2}\right) \mathrm{e}^{-\lambda\left(k_{\bar{m}+1, x}-k_{0, x}\right)}, k \in\left[k_{\bar{m}, x}, k_{\bar{m}+1, x}-1\right]$.

Note that from (13) and condition (ii), we have

$$
\begin{aligned}
V\left(k_{\bar{m}, x}\right) & =\sum_{i=1}^{N-1} \sum_{j=i+1}^{N} x_{i j}^{T}\left(k_{\bar{m}, x}-1\right)\left(I+J_{u}\left(k_{\bar{m}, x}\right)\right)^{T} \\
& \cdot P_{\sigma\left(k_{\bar{m}, x}\right)}\left(I+J_{u}\left(k_{\bar{m}, x}\right)\right) x_{i j}\left(k_{\bar{m}, x}-1\right) \\
\leq & \mu \lambda_{\max }^{2}\left(I+J_{u}\left(k_{\bar{m}, x}\right)\right) \\
& \cdot \sum_{i=1}^{N-1} \sum_{j=i+1}^{N} x_{i j}^{T}\left(k_{\bar{m}, x}-1\right) P_{\sigma\left(k_{\bar{m}, x}\right)} x_{i j}\left(k_{\bar{m}, x}-1\right) \\
& =\mu \lambda_{\max }^{2}\left(I+J_{u}\left(k_{\bar{m}, x}\right)\right) V\left(k_{\bar{m}, x}-1\right) \\
\leq & \mu \lambda_{\max }^{2}\left(I+J_{u}\left(k_{\bar{m}, x}\right)\right) M \varphi\left(\|\phi(\theta)\|_{\tau}^{2}\right) \mathrm{e}^{-\lambda\left(k_{\bar{m}, x}-k_{0, x}\right)} \\
& <\mu \lambda_{\max }^{2}\left(I+J_{u}\left(k_{\bar{m}, x}\right)\right) M \varphi\left(\|\phi(\theta)\|_{\tau}^{2}\right) \\
& \cdot \mathrm{e}^{-\lambda\left(k_{\bar{m}+1, x}-k_{0, x}\right)} \mathrm{e}^{\varepsilon_{\sigma\left(k_{\bar{m}, x}\right)}\left(k_{\bar{m}+1, x}-k_{\bar{m}, x}\right)} \\
\leq & M \varphi\left(\|\phi(\theta)\|_{\tau}^{2}\right) \mathrm{e}^{-\lambda\left(k_{\bar{m}+1, x}-k_{0, x}\right)} \mathrm{e}^{-\varepsilon_{\sigma\left(k_{\bar{m}, x}\right)}\left(k_{\bar{m}+1, x}-k_{\bar{m}, x}\right)}
\end{aligned}
$$


If (39) is not true, then there exists a natural number $\bar{k}$ satisfying

$$
\begin{gathered}
\bar{k}=\min \left\{k \in\left[k_{\bar{m}, x}, k_{\bar{m}+1, x}-1\right]: V(k)>\right. \\
\left.M \varphi\left(\|\phi(\theta)\|_{\tau}^{2}\right) \mathrm{e}^{-\lambda\left(k_{\bar{m}+1, x}-k_{0, x}\right)}\right\} .
\end{gathered}
$$

Consequently, we have

$\bar{k}>k_{\bar{m}, x}$ and $V(\bar{k})>V\left(k_{\bar{m}, x}\right)$.

Define

$$
\begin{aligned}
& k^{*}=\max \left\{k \in\left[k_{\bar{m}, x}, \bar{k}\right]: V(k) \leq\right. \\
& \left.M \varphi\left(\|\phi(\theta)\|_{\tau}^{2}\right) \mathrm{e}^{-\lambda\left(k_{\bar{m}+1, x}-k_{0, x}\right)} \mathrm{e}^{-\varepsilon_{\sigma\left(k_{\bar{m}, x}\right)}\left(k_{\bar{m}+1, x}-k_{\bar{m}, x}\right)}\right\} .
\end{aligned}
$$

Thus, there exists $k^{*}$ such that $k^{*}<\bar{k}$.

For any $k \in\left[k^{*}+1, \bar{k}\right]$, we have

$V(k)>M \varphi\left(\|\phi(\theta)\|_{\tau}^{2}\right) \cdot \mathrm{e}^{-\lambda\left(k_{\bar{m}+1, x}-k_{0, x}\right)} \mathrm{e}^{-\varepsilon_{\sigma\left(k_{\bar{m}, x}\right)}\left(k_{\bar{m}+1, x}-k_{\bar{m}, x}\right)}$,

and for any $k \in\left[k^{*}, \bar{k}\right]$,

$V\left(k^{*}\right) \leq V(k) \leq V(\bar{k})$.

Then, for any $k \in\left[k_{\bar{m}, x}, k_{\bar{m}+1, x}-1\right]$, we can obtain

$V(k+1) \leq\left[\frac{\lambda_{\max }\left(\Pi_{\sigma\left(k_{\bar{m}, x}\right)}\right)}{\lambda_{\min }\left(P_{\sigma\left(k_{\bar{m}, x}\right)}^{-1}\right)}+\mu q_{\sigma\left(k_{\bar{m}, x}\right)} \frac{\lambda_{\max }\left(\Omega_{\sigma\left(k_{\bar{m}, x}\right)}\right)}{\lambda_{\min }\left(P_{\sigma\left(k_{\bar{m}-m_{\tau}, x}\right)}^{-1}\right)}\right] V(k)$.

According to the condition (iii), one observes that for any $s \in \mathbb{Z}_{\tau}, k+s \in\left[k_{\bar{m}-m_{\tau}, x}, \bar{k}\right]$.

Meanwhile for any $k \in\left[k^{*}, \bar{k}-1\right]$,

$$
\begin{aligned}
V(k+s) & \leq M \varphi\left(\|\phi(\theta)\|_{\tau}^{2}\right) \mathrm{e}^{-\lambda\left(k_{\bar{m}-m_{\tau}+1, x}-k_{0, x}\right)} \\
& \leq M \varphi\left(\|\phi(\theta)\|_{\tau}^{2}\right) \mathrm{e}^{-\lambda\left(k_{\bar{m}+1, x}-k_{0, x}\right)} \\
& \cdot \mathrm{e}^{\sum_{i=0}^{m_{\tau}-1} \varepsilon_{\sigma\left(k_{\bar{m}-i, x}\right)}\left(k_{\bar{m}+1-i, x}-k_{\bar{m}-i, x}\right)} \\
& \leq \mathrm{e}^{-\varepsilon_{\sigma\left(k_{\bar{m}, x}\right)}\left(k_{\bar{m}+1, x}-k_{\bar{m}, x}\right)} M \varphi\left(\|\phi(\theta)\|_{\tau}^{2}\right) \mathrm{e}^{-\lambda\left(k_{\bar{m}+1, x}-k_{0, x}\right)} \\
& \cdot \mathrm{e}^{\varepsilon_{\sigma\left(k_{\bar{m}, x}\right)}\left(k_{\bar{m}+1, x}-k_{\bar{m}, x}\right)} \mathrm{e}^{\sum_{i=0}^{m_{\tau}-1} \varepsilon_{\sigma\left(k_{\bar{m}-i, x}\right)}\left(k_{\bar{m}+1-i, x}-k_{\bar{m}-i, x}\right)} \\
& \leq \mathrm{e}^{\varepsilon_{\sigma\left(k_{\bar{m}, x}\right)}\left(k_{\bar{m}+1, x}-k_{\bar{m}, x}\right)+\sum_{i=0}^{m_{\tau}-1} \varepsilon_{\sigma\left(k_{\bar{m}-i, x}\right)}\left(k_{\bar{m}+1-i, x}-k_{\bar{m}-i, x}\right)} \\
& \cdot V\left(k^{*}+1\right) \\
& <\mathrm{e}^{\varepsilon_{\sigma\left(k_{\bar{m}, x}\right)}\left(k_{\bar{m}+1, x}-k_{\bar{m}, x}+1\right)+\sum_{i=0}^{m_{\tau}-1} \varepsilon_{\sigma\left(k_{\bar{m}-i, x}\right)}\left(k_{\bar{m}+1-i, x}-k_{\bar{m}-i, x}\right)} \\
& \cdot V\left(k^{*}\right) \\
& \leq q_{\sigma\left(k_{\bar{m}, x}\right)} V(k) .
\end{aligned}
$$

Consider that $k=\bar{k}$, we have

$$
\begin{aligned}
V(\bar{k}) & \leq\left[\frac{\lambda_{\max }\left(\Pi_{\sigma\left(k_{\bar{m}, x}\right)}\right)}{\lambda_{\min }\left(P_{\sigma\left(k_{\bar{m}, x}\right)}^{-1}\right)}\right. \\
& \left.+\mu q_{\sigma\left(k_{\bar{m}, x}\right)} \frac{\lambda_{\max }\left(\Omega_{\sigma\left(k_{\bar{m}, x}\right)}\right)}{\lambda_{\min }\left(P_{\sigma\left(k_{\bar{m}-m_{\tau}, x}\right)}^{-1}\right)}\right]^{\left(\bar{k}-k^{*}\right)} V\left(k^{*}\right) \\
& \leq\left[\frac{\lambda_{\max }\left(\Pi_{\sigma\left(k_{\bar{m}, x}\right)}\right)}{\lambda_{\min }\left(P_{\sigma\left(k_{\bar{m}, x}\right)}^{-1}\right)}\right. \\
& \left.+\mu q_{\sigma\left(k_{\bar{m}, x}\right)} \frac{\lambda_{\max }\left(\Omega_{\sigma\left(k_{\bar{m}, x}\right)}\right)}{\lambda_{\min }\left(P_{\sigma\left(k_{\bar{m}-m_{\tau}, x}\right)}^{-1}\right.}\right]^{\left(k_{\bar{m}+1, x}-k_{\bar{m}, x}\right)} \\
& \cdot M \varphi\left(\|\phi(\theta)\|_{\tau}^{2}\right) \mathrm{e}^{-\lambda\left(k_{\bar{m}+1, x}-k_{0, x}\right)} \mathrm{e}^{-\varepsilon_{\sigma\left(k_{\bar{m}, x}\right)}\left(k_{\bar{m}+1, x}-k_{\bar{m}, x}\right)} \\
& <M \varphi\left(\|\phi(\theta)\|_{\tau}^{2}\right) \mathrm{e}^{-\lambda\left(k_{\bar{m}+1, x}-k_{0, x}\right)} \\
& <V(\bar{k})
\end{aligned}
$$

which is a contradiction. Hence, the Eq. (39) holds for $k=\bar{k}+1$. And by virtue of mathematical induction, the claim (18) is true for each $k \in \mathbb{N}$.

In view of (18) and Definition 1, it can be obtained that $V(k) \leq M \varphi\left(\|\phi(\theta)\|_{\tau}^{2}\right) \mathrm{e}^{-\lambda\left(k-k_{0, x}\right)}, k \in\left[k_{m-1, x}, k_{m, x}-1\right], m \in \mathbb{N}$.

For any $k \in \mathbb{N}$,

$$
\begin{aligned}
& \min \left\{\lambda_{\min }\left(P_{\sigma(k)}\right)\right\} \sum_{i=1}^{N-1} \sum_{j=i+1}^{N}\left\|x_{i}(k)-x_{j}(k)\right\|^{2} \\
& \leq \sum_{i=1}^{N-1} \sum_{j=i+1}^{N}\left(x_{i}(k)-x_{j}(k)\right)^{T} P_{\sigma(k)}\left(x_{i}(k)-x_{j}(k)\right) \\
& =V(k) \\
& \leq M \varphi\left(\|\phi(\theta)\|_{\tau}^{2}\right) \mathrm{e}^{-\lambda\left(k-k_{0, x}\right)}
\end{aligned}
$$

Therefor, for any $k \in \mathbb{N}$,

$$
\begin{aligned}
& \sum_{i=1}^{N-1} \sum_{j=i+1}^{N}\left\|x_{i}(k)-x_{j}(k)\right\|^{2} \\
& \leq \min \left\{\lambda_{\min }^{-1}\left(P_{\sigma(k)}\right)\right\} M \varphi\left(\|\phi(\theta)\|_{\tau}^{2}\right) \mathrm{e}^{-\lambda\left(k-k_{0, x}\right)},
\end{aligned}
$$

which implies

$\left\|x_{i}(k)-x_{j}(k)\right\| \leq M_{0} \mathrm{e}^{-\lambda\left(k-k_{0, x}\right)}, \quad 1 \leq i \leq j \leq N$.

Therefore, the discrete complex networks (1) are globally exponentially synchronized under impulsive control. The proof is thus completed.

Remark. We consider a multiple Lyapunov function for each sub-network with arbitrarily fast switching signal in our theorem, which results in a less conservation criterion.

Remark 1 In the switched Lyapunov function, $p_{\sigma(k)}$ gives an upper bound on the estimation of divergence rate for 
each running sub-network. By condition (ii) of Theorem 1, the impulsive control gain is designed to compensate divergence from system itself and deteriorating effect from arbitrarily fast switching. If some certain sub-networks could be self-synchronizing, the impulsive control gain only needs to compensate deteriorating effect.

\section{Example and numerical simulations}

This section presents a typical example to illustrate our result. Let us consider a 2-dimensional discrete chaotic neural network is given as the isolated node of a smallworld network with 30 nodes,

$x(k+1)=A x(k)+B f(x(k))+D f(x(k-\tau(k)))+I(k)$,
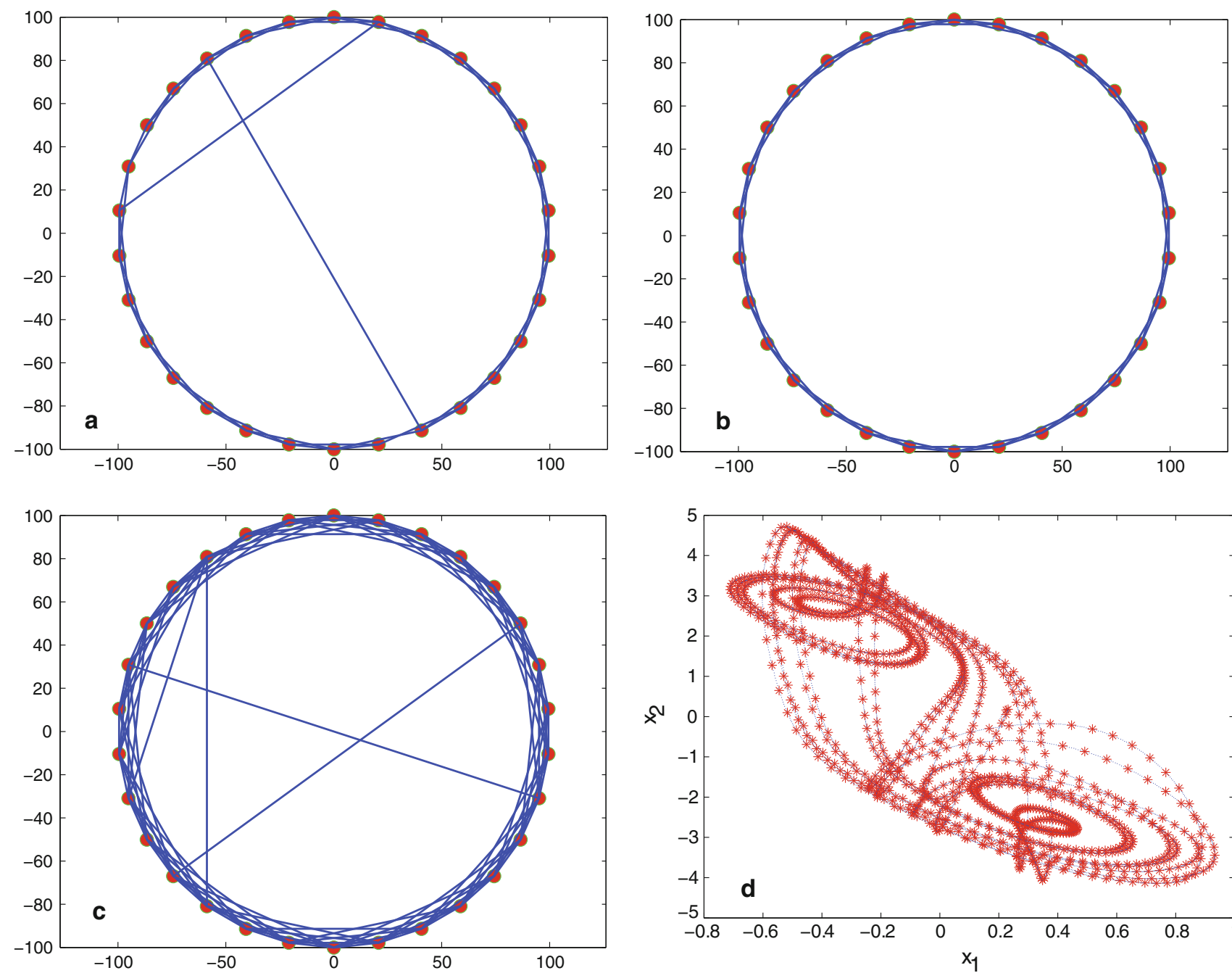

Fig. 1 a $N=30, k=2, p=0.02 ; \mathbf{b} N=30, k=2, p=0.01 ; \mathbf{c} N=30, k=4, p=0.04$; $\mathbf{d}$ Chaotic trajectory of each single node 
- 30 nodes are arranged in a ring, while each node $i$ is adjacent to its 2 neighbor node; each pair of nodes are coupled to the whole network by probability $p=0.04$. See Fig. 1c.

The trajectory of each single node of this small-world model has been portrayed in Fig. 1d with random initial values in the interval $[0.3,3]$ and $[-3,-0.3]$, respectively.

Let $\Gamma=\operatorname{diag}\{0.15,0.3\}$, we can obtain the state response of each sub-network. Note that there is no switched rule in each sub-network here, and the initial values are randomly chosen in the interval $[0.3,3]$ and $[-3$, $-0.3]$, respectively.

Given a switching signal $\sigma(t)$ in Fig. 3a, we have the state response of the switched complex networks, see Fig. 3b. From Theorem 1, for each sub-network, we have

$$
\begin{aligned}
& -P_{1}=\left[\begin{array}{cc}
3.0001 & 0 \\
0 & 3.0001
\end{array}\right], Q_{1,1}=\left[\begin{array}{cc}
5.0690 & 0 \\
0 & 5.0690
\end{array}\right], \quad Q_{2,1}= \\
& {\left[\begin{array}{cc}
9.3812 & 0 \\
0 & 9.3812
\end{array}\right], \quad Q_{3,1}=\left[\begin{array}{cc}
33.7971 & 0 \\
0 & 33.7971
\end{array}\right], \quad Q_{4,1}=} \\
& {\left[\begin{array}{cc}
11.4594 & 0 \\
0 & 11.4594
\end{array}\right], Q_{5,1}=\left[\begin{array}{cc}
50.9300 & 0 \\
0 & 50.9300
\end{array}\right], Q_{6,1}=} \\
& {\left[\begin{array}{cc}
20.1879 & 0 \\
0 & 20.1879
\end{array}\right] \text {. And } \varepsilon_{1}=0.6213, q_{1}<22.42 \text {. Thus, }} \\
& J_{1}=\left[\begin{array}{cc}
-0.6667 & 0 \\
0 & -0.667
\end{array}\right] \text {. } \\
& \text { - } P_{2}=\left[\begin{array}{cc}
2.9998 & 0 \\
0 & 2.9998
\end{array}\right], Q_{1,2}=\left[\begin{array}{cc}
5.1333 & 0 \\
0 & 5.1333
\end{array}\right], Q_{2,2}= \\
& {\left[\begin{array}{cc}
9.9398 & 0 \\
0 & 9.9398
\end{array}\right], \quad Q_{3,2}=\left[\begin{array}{cc}
29.7933 & 0 \\
0 & 29.7933
\end{array}\right], \quad Q_{4,2}=} \\
& {\left[\begin{array}{cc}
11.2764 & 0 \\
0 & 11.2764
\end{array}\right], \quad Q_{5,2}=\left[\begin{array}{cc}
52.0308 & 0 \\
0 & 52.0308
\end{array}\right] \text {, }}
\end{aligned}
$$
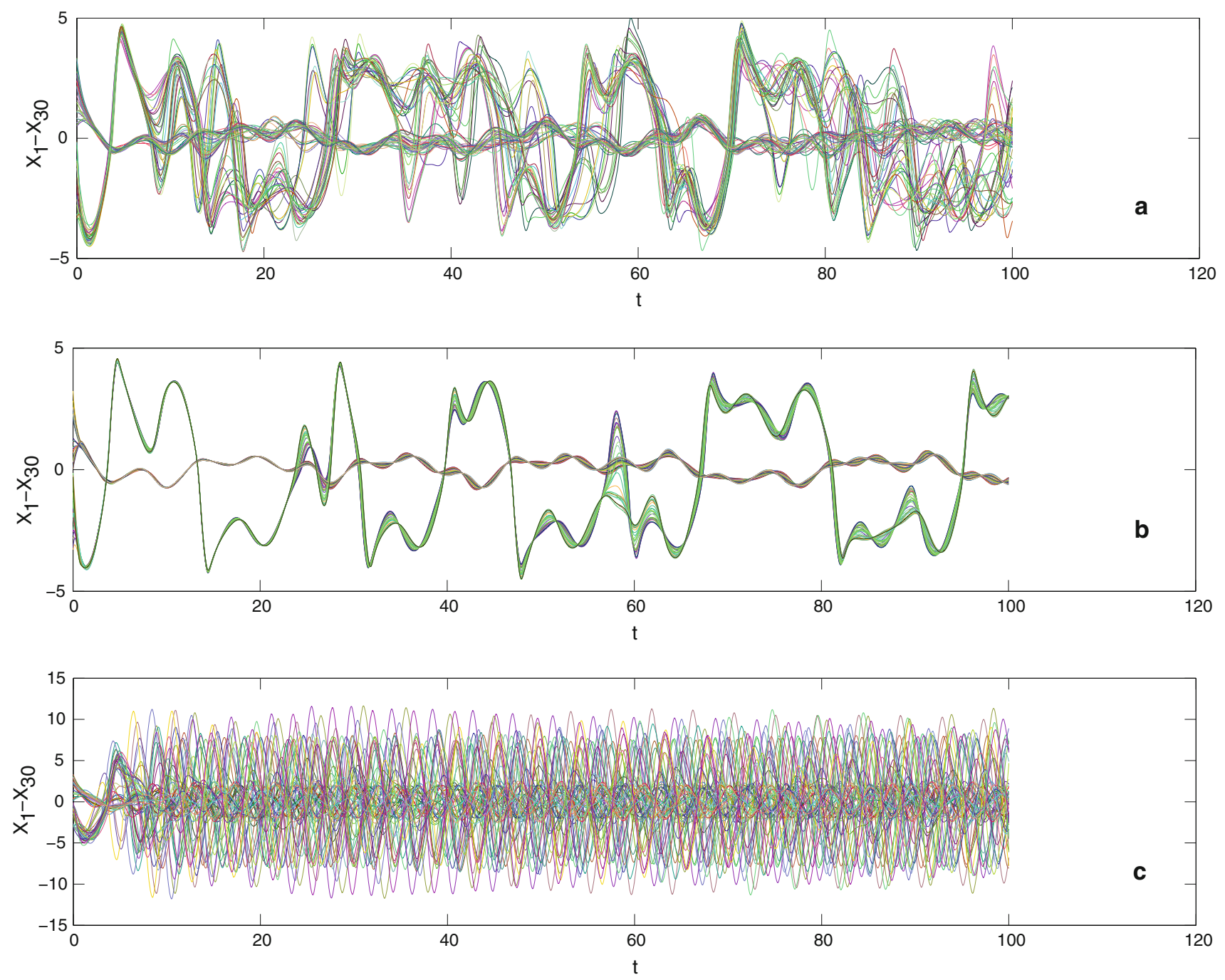

Fig. 2 a The state responses of sub-networks 1; b the state responses of sub-networks 2; $\mathbf{c}$ the state responses of sub-networks 3 


$$
\begin{aligned}
& Q_{6,2}=\left[\begin{array}{cc}
19.9970 & 0 \\
0 & 19.9970
\end{array}\right] . \text { And } \varepsilon_{2}=0.8870, q_{2}<4.22 . \\
& \text { Thus, } J_{2}=\left[\begin{array}{cc}
-0.4079 & 0 \\
0 & -0.4079
\end{array}\right] . \\
&-P_{3}=\left[\begin{array}{cc}
13.4267 & 0 \\
0 & 13.4267
\end{array}\right], Q_{1,3}=\left[\begin{array}{cc}
10.2609 & 0 \\
0 & 10.2609
\end{array}\right], \\
& Q_{2,3}=\left[\begin{array}{cc}
3.1001 & 0 \\
0 & 3.1001
\end{array}\right], Q_{3,3}=\left[\begin{array}{cc}
16.6667 & 0 \\
0 & 16.6667
\end{array}\right], \\
& Q_{4,3}=\left[\begin{array}{cc}
5.2060 & 0 \\
0 & 5.2060
\end{array}\right], Q_{5,3}=\left[\begin{array}{cc}
30.8123 & 0 \\
0 & 30.8123
\end{array}\right], \\
& Q_{6,3}=\left[\begin{array}{cc}
3.0112 & 0 \\
0 & 3.0112
\end{array}\right] . \text { And } \varepsilon_{3}=0.1062, q_{3}<12.62 . \\
& \text { Thus, } J_{3}=\left[\begin{array}{cc}
-1.1576 & 0 \\
0 & -1.1576
\end{array}\right] .
\end{aligned}
$$

It is shown from Fig. 2a-c that all of nodes in each subnetwork could not reach into a synchronous state without a control. Indeed, the switched signal plays a role of deterioration accelerator to diverge the synchronous state, shown in Fig. 3b. Once the feasible impulsive controller is placed on discrete complex networks with topology switching, such complex networks would be synchronized, see Fig. 3c.

\section{Conclusion}

In this paper, we have investigated impulsive synchronization control of a discrete delayed complex network with switching topology by using Lyapunov-Ruzimiki method. A time-varying delay-dependent criteria for exponential synchronization is presented guarantee the switched discrete complex networks tending to be a synchronous manifold. It is worthwhile to see time-varying delay can take any value, even larger than any dwell time of a subnetwork. Furthermore, a numerical example with three subnetworks is presented by using the impulsive control technique.
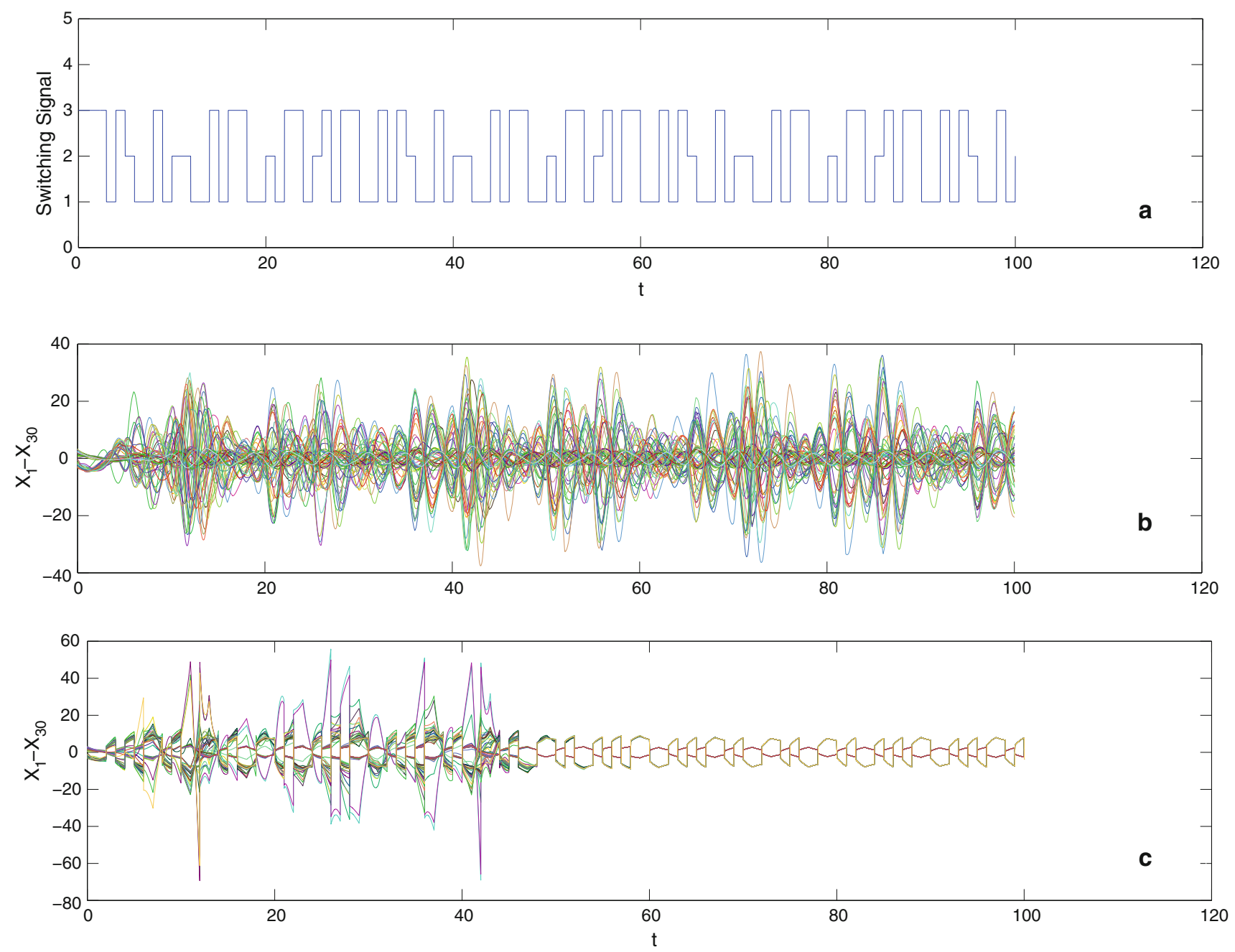

Fig. 3 a The switching signal $\sigma(\mathrm{t})$; $\mathbf{b}$ the state responses of the switched system; $\mathbf{c}$ the synchronized state under impulsive control 
Acknowledgments This research is supported by Australia Government grant through the Collaborative Research Network (CRN) to the university of Ballarat.

Open Access This article is distributed under the terms of the Creative Commons Attribution License which permits any use, distribution, and reproduction in any medium, provided the original author(s) and the source are credited.

\section{References}

1. Watts DJ, Strogatz SH (1998) Collective dynamics of 'smallworld' networks. Nature 393:440-442

2. Barabási AL, Albert R (1999) Emergence of scaling in random networks. Science 286:509-512

3. Strogatz SH (2001) Exploring complex networks. Nature 410:268-276

4. VanWiggeren GD, Roy R (1998) Communication with chaotic lasers. Science 279:1198-1200

5. Fischer I, Liu Y, Davis P (2000) Synchronization of chaotic semiconductor laser dynamics on subnanosecond time scales and its potential for chaos communication. Phys Rev A 62:011801$1-011801-4$

6. Hoppensteadt FC, Izhikevich EM (2000) Pattern recognition via synchronization in phase-locked loop neural networks. IEEE Trans Neural Netw 11:734-738

7. Wang XF, Chen GR (2002) Synchronization in scale-free dynamical networks:robustness and fragility. IEEE Trans Circuits Syst I, Reg Papers 49:54-62

8. Wu C (2005) Synchronization in networks of nonlinear dynamical systems coupled via a directed graph. Nonlinearity 18:1057-1064

9. Wang Z, Wang Y, Liu Y (2010) Global synchronization for discrete-time stochastic complex networks with randomly occurred nonlinearities and mixed time delays. IEEE Trans Neural Netw 21:11-25

10. Li X, Wang XF, Chen G (2004) Pinning a complex network to its equilibrium. IEEE Trans Circuits Syst I Reg Papers 51:2074-2087

11. Zhou J, Lu J, Lü J (2006) Adaptive synchronization of an uncertain complex dynamical networks. IEEE Trans Autom Control 51:652-656

12. Chen T, Liu X, Lu W (2007) Pinning complex networks by a single controller. IEEE Trans Circuits Syst I Reg Papers 54:1317-1326

13. Lu W, Chen T (2004) Synchronization analysis of linearly coupled networks of discrete time systems. Phys D 198:148-168

14. Ribeiro A, Zhu R, Kauffman SA (2006) A general modeling strategy for gene regulatory networks with stochastic dynamics. J Comput Biol 13:1630-1639

15. Yao J, Wang HO, Guan ZH, Xu W (2009) Passive stability and synchronization of complex spatio-temporal switching networks with time delays. Automatica 45:1721-1728

16. Atay FM, Biyikoglu T, Jost J (2006) Network synchronization: spectral versus statistical properties. Phys D 224:35-41

17. Belykh IV, Belykh VN, Hasler M (2004) Blinking model and synchronization in small-world networks with a time-varying coupling. Phys D 195:188-206
18. Wu CW, Chua LO (1995) Synchronization in an array of linearly coupled dynamical systems. IEEE Trans Circuits Syst I Reg Papers 42:430-447

19. Barahona M, Pecora LM (2002) Synchronization in small-world systems. Phys Rev Lett 89:054101

20. Lu W, Atay FM, Jost J (2007) Synchronization of discrete-time networks with time-varying couplings. SIAM J Math Anal 39:1231-1259

21. Lu J, Ho DWC, Wu L (2009) Exponential stabilization in switched stochastic dynamical networks. Nonlinearity 22:889-911

22. Zhou J, Wu Q, Xiang L (2011) Pinning complex delayed dynamical networks by a single impulsive controller. IEEE Trans Circuits Syst I Reg Papers 58:2882-2893

23. Lu J, Ho DWC, Wang ZD (2009) Pinning stabilization of linearly coupled stochastic neural networks via minimum number of controllers. IEEE Trans Neural Netw 20:1617-1629

24. Lu J, Kurths J, Cao JD, Mahdavi N, Huang C (2012) Synchronization control for nonlinear stochastic dynamical networks: pinning impulsive strategy. IEEE Trans Neural Netw Learn Syst 23:285-292

25. Huang T, Chen G, Kurth J (2011) Synchronization of chaotic systems with time-varying coupling delays. Discrete Contin Dyn Syst Ser B 16:1071-1082

26. Huang T, Li C, Gao D, Xiao M (2012) Anticipating synchronization through optimal feedback control. J Global Optim $52: 281-290$

27. Wen S, Zeng Z, Huang T (2012) Adaptive synchronization of memristor-based Chua's circuits. Phys Lett A 376:2775-2780

28. Wen S, Zeng Z, Huang T (2012) H-infinity filtering for neutral systems with mixed delays and multiplicative noises. IEEE Trans Circuits Syst Part II 59(11):820-824

29. Wen S, Zeng Z, Huang T (2013) Robust probabilistic sampling $H$ output tracking control for a class of nonlinear networked systems with multiplicative noises. J Franklin Inst 350:1093-1111

30. Yang $T$ (2001) Impulsive systems and control: theory and applications. Nova Science, Huntington, NY

31. Li CD, Shen YY, Feng G (2008) Stabilizing effects of impulse in delayed BAM neural networks. IEEE Trans Circuits Syst II Brief Papers 53:1284-1288

32. Li CJ, Li CD, Liao XF, Huang TW (2011) Impulsive effects on stability of high-order BAM neural networks with time delays. Neurocomputing 74:1541-1550

33. Li CJ, Li CD, Huang TW (2011) Exponential stability of impulsive high order Hopfield-type neural networks with delays and reaction-diffusion. Int J Comput Math 88:3150-3162

34. Song QK, Cao J (2007) Stability analysis of impulsive cohengrossberg neural network with unbounded discrete time-varying delays. Int J Neural Syst 17(5):407-417

35. Song QK, Wang Z (2008) Stability analysis of impulsive stochastic Cohen-Grossberg neural networks with mixed time delays. Phys A 387(13):3314-3326

36. Song QK, Cao J (2007) Impulsive effects on stability of fuzzy Cohen-Grossberg neural networks with time-varying delays. IEEE Trans Syst Man Cybern B Cybern 37(3):733-741

37. Song QK, Zhang JY (2008) Global exponential stability of impulsive Cohen-Grossberg neural network with time-varying delays. Nonlinear Anal Real World Appl 9(2):500-510

38. Langville AN, Stewart WJ (2004) The Kronecker product and stochastic automata networks. J Comput Appl Math 167:429-447 